International Journal of Engineering \& Technology, $7(2.21)(2018) 391-393$
International Journal of Engineering \& Technology
SPC
Website: www.sciencepubco.com/index.php/IJET
Research paper

\title{
IOT connected predictive vehicle systems
}

\author{
Murugadass $^{*}$, P. Sheela Gowr ${ }^{2}$, M. Latha ${ }^{3}$, U.V. Anbazhagu ${ }^{4}$ \\ ${ }^{1}$ Department of Computer Science and Engineering, Vels Institute of Science, Technology and \\ Advanced Studies (VISTAS), Chennai, Tamilnadu, India. \\ ${ }^{2}$ Department of Computer Science and Engineering, Vels Institute of Science, Technology and \\ Advanced Studies (VISTAS), Chennai, Tamilnadu, India. \\ ${ }^{3}$ Department of Computer Science and Engineering, Vels Institute of Science, Technology and \\ Advanced Studies (VISTAS), Chennai, Tamilnadu, India. \\ ${ }^{4}$ Department of Computer Science and Engineering, Vels Institute of Science, Technology and \\ Advanced Studies (VISTAS), Chennai, Tamilnadu, India. \\ *Corresponding author E-mail: sridharanbe.ece@gmail.com
}

\begin{abstract}
Predictive maintenance is to identify vehicle maintenance issues before they occur. By leveraging data from navigation locator and motion of vehicle, status and parts of the vehicle, requirement of service, warranty repairs with current vehicle sensor data would be difficult for a human to discover. Predictive data analytics can find meaningful correlations via Connected Vehicle which is a technological advancement in Automobile industry. Using Internet of Things IOT, various information like health information of a driving person and navigation of vehicle can be easily monitored. Connected vehicle deals with cars and other vehicles where we the data will be shared with the backed applications like micro services.
\end{abstract}

Keywords: Internet of things, connected cars, predictive maintenance, MQTT, eclipse mosquito, spring tool suite, Smart City.

\section{Introduction}

This project deals with the predictive vehicle systems from which it is possible to manage and maintain the car internally as well it controls the number of accidents which is happening in the current world scenario. This concern has been achieved by the technology called Internet of Things which is the most required platform in information technology and communication systems. From this we can be able to achieve all the goals as per the end user needs.

\subsection{Periodic maintenance of vehicle}

In this periodic maintenance, the end user has to spend amount of money for the service as well as the infrastructure for the vehicle. And also they need to spend lot of time for maintenance service of the vehicle.

\subsection{Drawbacks of periodic maintenance}

This periodic maintenance leads to high cost of service and the parts modification and also they have to follow the time period for each and every maintenance services.

\section{Proposed model}

In this proposed Model we can stop looking at the time for the vehicle care and maintenance. Because it will be taken care by the intelligent software and the hardware, which is going to connected to the vehicle on load systems using internet of things.

Advantages of Proposed Model - It reduces the time and cost of the vehicle which compares to the existing periodic maintenance.
And also it controls the vehicle accidents because it can navigate the vehicle in the right path. When the most accidental zone comes it will make the intimation calls to the driver who is handling the vehicle.

\section{Model architecture}

The Proposed Model architecture is entirely based on the Internet of things technology from which the intelligent software is embedded into the hardware system which is loaded in the vehicle which is shown in Figure 1. Open Source Tools Used For The Model - Spring Tools Suite for writing micro services based development modules, Apache maven for building the codes, Docker Containers for the deployment mode. Apache Kafka for the Messaging Systems and its architecture has been shown in Figure 3.

$$
\text { Car } \ll \rightarrow \text { Service Provider } \leftarrow \rightarrow \text { Drivers }
$$

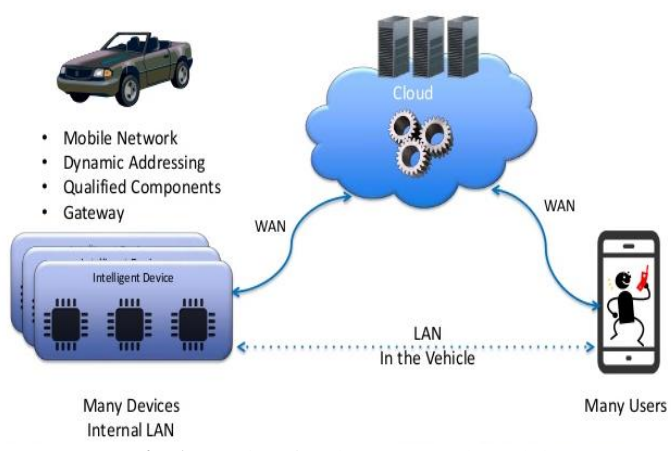

Fig.1: Design for the proposed model 


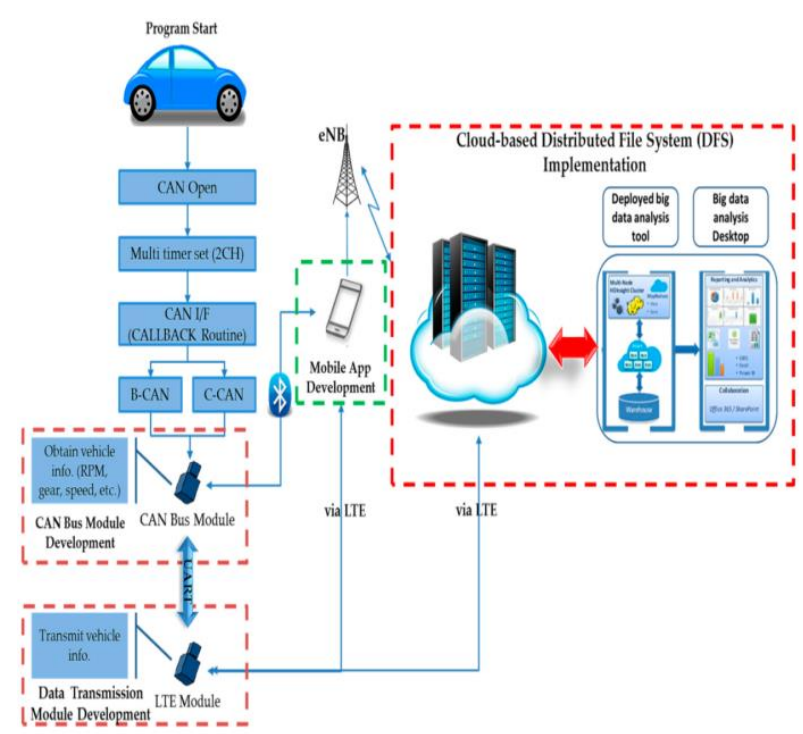

Fig.2: Architecture of the model

\subsection{Overview of the architecture}

1. The architecture is based on the CAN protocol / RashberryPi which is installed in the On loading systems of the vehicle.

2. A protocol has been connected with the LTE module which is connected in the driver's cabinet of the vehicle.

3. LTE is supposed to be installed in the Driver's SIM card of any service providers for the internet connection.

4. Once the internet connectivity has been done, the software has to be installed in mobile or can be viewed in web for interacting between the vehicle systems.

5. The installed software can be accessed from anywhere because it's already deployed in cloud. So the software will pick all the data's from the service which has been exposed by the rest API's in cloud platforms.

6. Architecture for the system has been shown in Figure 2.

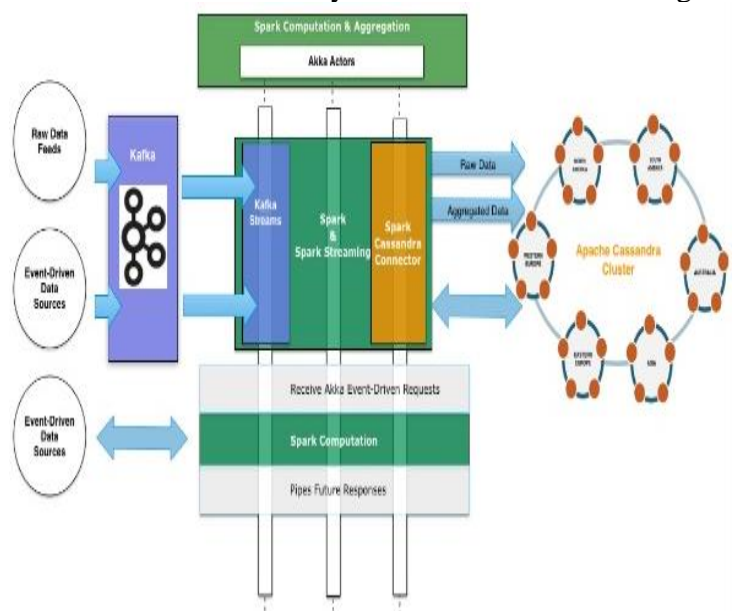

Fig.3: Kafka messaging system architecture

\section{Cloud setup architecture}

The Cloud deployment has been achieved using the heroku cloud since it has easy integration with the cloud repository like GitHub, bitbucket etc. As per that all micro services components has been checked in with the Cloud Repository and then it has been configured to the heroku server in cloud. Whenever any new update or changes comes to the existing system, the heroku will enable the auto deployment services. The deployment architecture has to be followed using the heroku cloud deployments. Since the services are platform independent it can be deployed in any type of cloud servers.

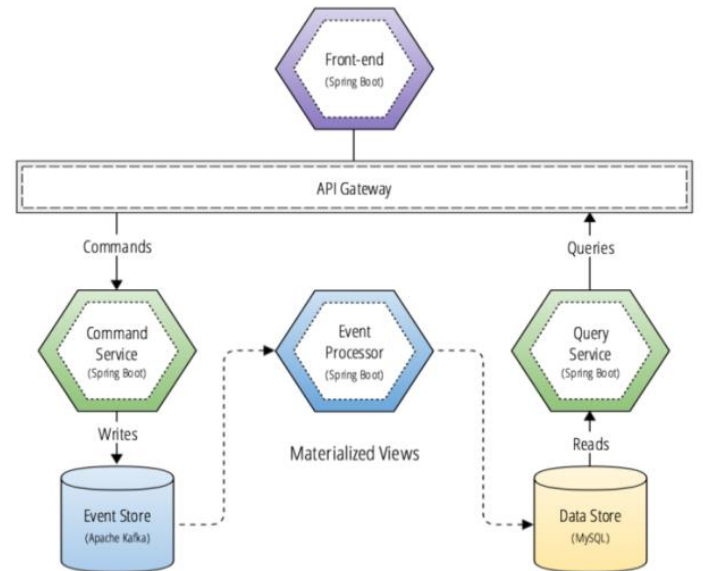

Sample output of the predictive systems

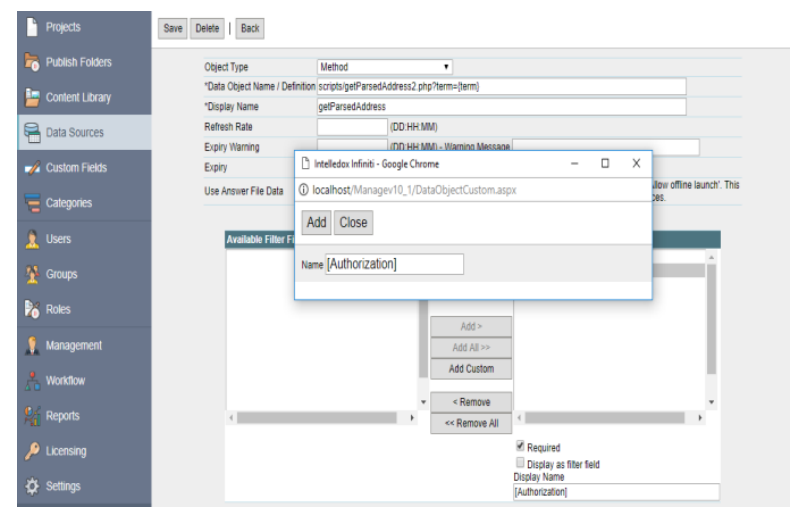

Mobile App Sample Outputs
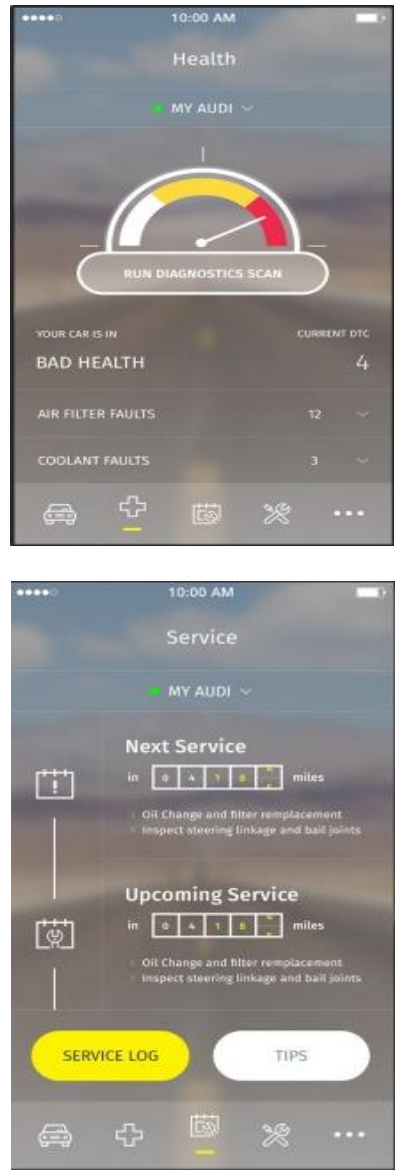


\section{Conclusion}

The proposed predictive model has been achieved by using all the technologies which has defined above and also still it has many challenges in the current vehicle systems in the world. A Raspberry Pi3 interface links with CAN (Controller Aided Network) has been implemented which generates dynamic vehicle data helping the driver to get updated information about his vehicle and health information. It shows the entire information about the vehicle and its navigation, thus providing more security to the driver and helps to manage traffic easily preventing accidents.

\section{References}

[1] SAP/iot-starter kit. GitHub based on Raspberry Pi - Teach, Learn, and Make with Raspberry Pi.

[2] The simplest way to experience IoT in the HANA Cloud PlatformPart 1 by Jeff Durnwald based on Simple link SensorTag - TI.com

[3] Measure the Sun with Hana Cloud Platform - Hands On Tutorial 2.0 by Marcus Conrad Behrens based on Electric Imp

[4] RaspberryPi on SAP HANA Cloud Platform by Rui Nogueira base on Raspberry Pi - Teach, Learn, and Make with Raspberry Pi

[5] Hand's-On Video Tutorials for Internet of Things (IoT) Services by Philip MUGGLESTONE based on Simple link SensorTag - TI.com

[6] SAP HANA IoT Part 1: Introduction to Arduino, Raspberry Pi and why we have selected them by Ajay Nayak.

[7] Particle Photon or Core on HCP - IoT by A. Kamhoot based on Particle (formerly Spark) | Prototyping tools for the Internet of Things.

[8] Connecting mobile devices with IFTTT and Zapier to SAP HCP IoT Service by Weber based on IFTTT. 